\title{
Viabilidade de uso de imagens do Landsat em mapeamento de área cultivada com soja no Estado do Paraná
}

\author{
Luciana Miura Sugawara(1), Bernardo Friedrich Theodor Rudorff(1) e Marcos Adami(1)
}

\begin{abstract}
(1)Instituto Nacional de Pesquisas Espaciais, Caixa Postal 515, CEP 12245-970 São José dos Campos, SP. E-mail: Imiura@dsr.inpe.br, bernardo@dsr.inpe.br, adami@dsr.inpe.br
\end{abstract}

\begin{abstract}
Resumo - O objetivo deste trabalho foi avaliar a viabilidade do uso de imagens do Landsat, para o mapeamento da área cultivada com soja, nas safras de 2000/2001 a 2006/2007, no Estado do Paraná. A análise dos "quick looks" das imagens dos sensores TM e ETM+ foi feita para selecionar as imagens úteis para o mapeamento da cultura da soja. Os "quick looks" foram classificados de acordo com a presença ou a ausência de nuvens e de problemas técnicos. Conforme os resultados, em nenhum dos sete anos teria sido possível mapear a área cultivada com soja, em todo o Estado, mesmo nos três anos-safra em que os satélites Landsat 5 e 7 operaram em conjunto. A presença de nuvens, detectada pelos sensores ópticos, deve ser levada em conta no mapeamento sistemático da área cultivada com culturas de verão, no Brasil.
\end{abstract}

Termos para indexação: Glycine max, cobertura de nuvens, resolução temporal, sensoriamento remoto óptico.

\section{Feasibility of the use of Landsat imagery to map soybean crop areas in Paraná, Brazil}

\begin{abstract}
The aim of this work was to evaluate the feasibility of the use of Landsat imagery to map soybean crop areas in Paraná, Brazil, during crop years from 2000/2001 to 2006/2007. The analysis of the quick look images from the TM and ETM+ sensors was performed to select useful images to map soybean crop. The quick looks were classified according to the presence or absence of clouds and technical problems. It was verified that for none of the seven crop years it would have been possible to map soybean crop for the entire Paraná state, even for the three crop years during which both satellites Landsat 5 and 7 were operating simultaneously. The presence of clouds, detected through the optical sensors, should be considered for systematic mapping of summer crops in Brazil.
\end{abstract}

Index terms: Glycine max, cloud cover, temporal resolution, optical remote sensing.

\section{Introdução}

Durante muito tempo as imagens de sensoriamento remoto (SR) foram apresentadas como ferramenta de grande potencial para diversas aplicações na agricultura (Mendonça et al., 1979). Apesar de vários trabalhos demonstrarem esse potencial em pequenas áreas (Oetter et al., 2000; Ippoliti-Ramilo et al., 2003; Doraiswamy et al., 2004; Maxwell et al., 2004; Van Niel \& Mcvicar, 2004; Lamparelli et al., 2008), poucos trabalhos foram feitos com o uso das imagens com resolução espacial média (30 a $50 \mathrm{~m}$ ), no mapeamento de culturas agrícolas, em grandes áreas no Brasil. Exemplos desse tipo de mapeamento podem ser vistos nos trabalhos de Fontana et al. (2000), Rizzi \& Rudorff (2005) e Rudorff \& Sugawara (2007), que relatam que a freqüente cobertura de nuvens nas imagens obtidas pelos satélites da série Landsat dificulta e até mesmo impede que elas sejam utilizadas para fins de mapeamento e estimativa de área cultivada de culturas agrícolas no Brasil, particularmente as de verão. Além disso, no caso da soja, é fundamental que as imagens sejam analisadas em mais de um período específico dentro da estação de crescimento da cultura a fim de que ela seja identificada e mapeada corretamente (Fontana et al., 2000; Berka \& Rudorff, 2003; Van Niel \& Mcvicar, 2004; Rizzi \& Rudorff, 2005).

Essa dificuldade pode ser contornada com o aumento da resolução temporal ou com o uso de imagens obtidas por diferentes satélites e/ou sensores, a fim de aumentar a probabilidade de aquisição de imagens livres de cobertura de nuvens (Wulder et al., 2008a). O aumento da resolução temporal também pode ser proporcionado por constelações de satélites (Dorigo et al., 2007; Wulder et al., 2008b) ou, por exemplo, pela utilização de imagens quase diárias, adquiridas pelo sensor "moderate resolution imaging spectroradiometer" (MODIS), a bordo da plataforma Terra (Justice \& Townshend, 2002). 
No Brasil, até o momento não se tem conhecimento do uso operacional das imagens de SR para o mapeamento sistemático e anual de uma grande cultura de verão em escala regional ou nacional. Um exemplo do uso de imagens de SR para a estimativa de área cultivada com cana-de-açúcar é o projeto Canasat, que desde 2003 vem sistematicamentemapeandoas lavouras de cana-de-açúcar no Estado de São Paulo e, desde 2005, nos demais estados da região Centro-Sul. Esse projeto fornece a estimativa da área de cana-de-açúcar disponível para colheita antes do início de cada ano safra (Rudorff \& Sugawara, 2007).

O contexto histórico apresentado mostra que, apesar do potencial das imagens de SR óptico para a estimativa da área plantada de culturas agrícolas, existem limitações para o seu uso operacional. Essas limitações têm sido pouco investigadas, já que, de acordo com Sano et al. (2007), a disponibilidade espacial e temporal de imagens semelhantes aos satélites da série Landsat é pouco conhecida. Isto dificulta a especificação de missões de SR para tais aplicações.

Asner (2001) e Sano et al. (2007) analisaram a probabilidade de ocorrência de nuvens nas imagens do satélite Landsat na Amazônia brasileira e no Cerrado, respectivamente. Os autores mostraram que o acompanhamento sazonal dessas áreas, principalmente no verão, é dificultado pela alta ocorrência de nuvens nas imagens. Ju \& Roy (2008) apontaram a dificuldade de obtenção de imagens livres de nuvens sazonalmente em escala mundial.

Com o intuito de abordar as questões relacionadas com a cobertura de nuvens e a aquisição das imagens dos sensores "thematic mapper" (TM) e "enhanced thematic mapper plus" $(\mathrm{ETM}+)$ - a bordo dos satélites Landsat 5 e 7, respectivamente - este trabalho teve o objetivo de avaliar, ao longo de sete anos safra (2000/2001 a 2006/2007), a disponibilidade dessas imagens para a identificação e o mapeamento da área cultivada com soja no Estado do Paraná.

\section{Material e Métodos}

A área de estudo compreende o Estado do Paraná, na região Sul do Brasil, localizado entre $22^{\circ} 29^{\prime} \mathrm{S}$ e $26^{\circ} 43^{\prime} \mathrm{S}$ e $48^{\circ} 2^{\prime} \mathrm{W}$ e $54^{\circ} 38^{\prime} \mathrm{W}$, conforme a Figura 1 (Paraná, 1987). Nesta Figura também está apresentada a estratificação da área cultivada com soja, por município, no Estado do Paraná, bem com a abrangência das cenas e suas respectivas órbitas e pontos no sistema Landsat de referência universal "worldwide reference system" WRS. Com base nesses dados, verificou-se que o cultivo

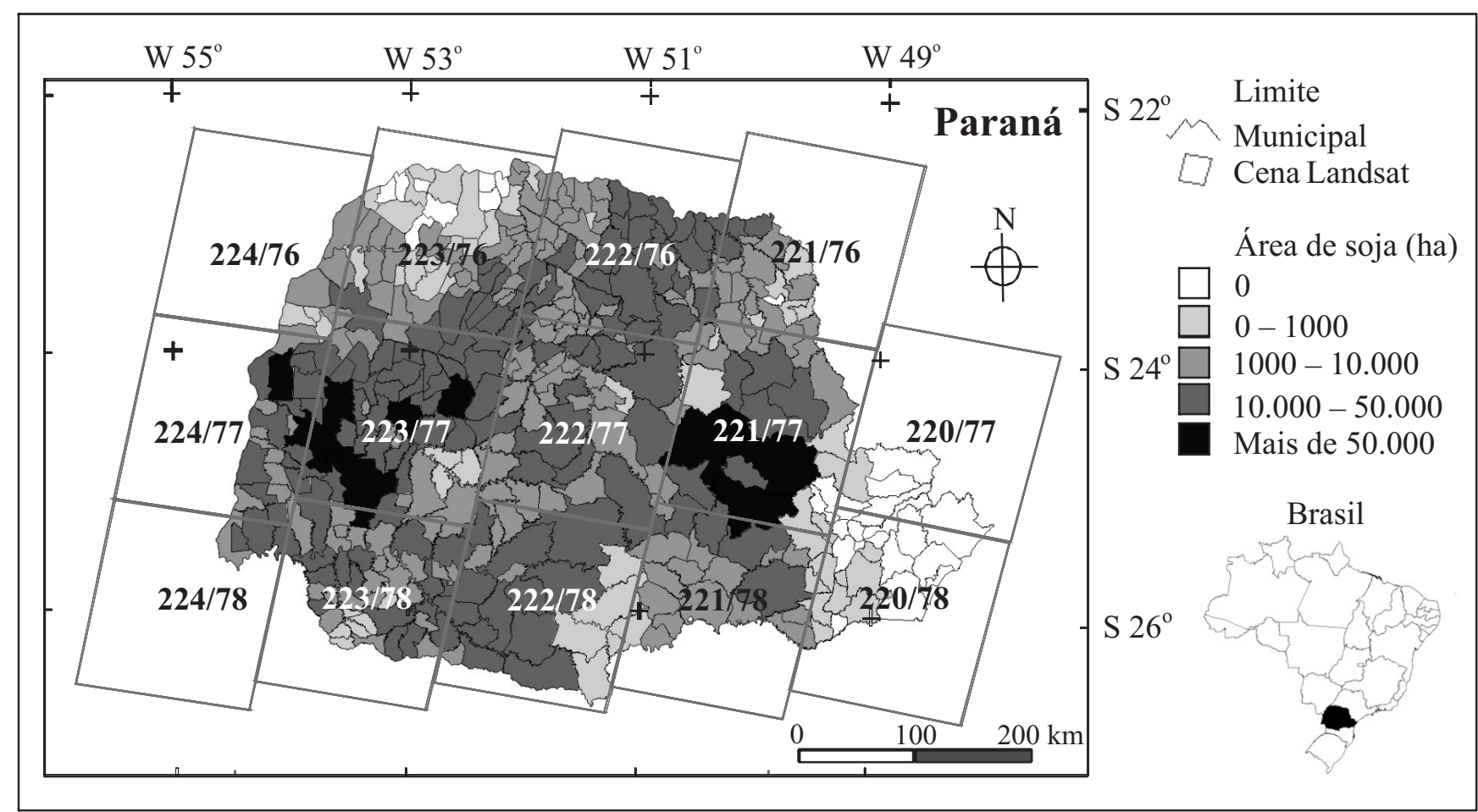

Figura 1. Localização da abrangência das cenas das imagens por órbita e ponto do sistema Landsat de referência universal sobre a malha municipal e a área de soja, por município, do Estado do Paraná. Fonte: adaptada de IBGE (2007a, 2007b) e United States Geological Survey (2008). 
da soja ocorre em todas as cenas do Landsat que cobrem o Estado do Paraná.

O estudo, para avaliar a disponibilidade de imagens para o mapeamento da área cultivada de soja, foi realizado nos anos-safra de 2000/2001 a 2006/2007. Neste período foram analisados todos os "quick looks" - imagem em baixa resolução que permite avaliar a qualidade das imagens de forma visual - das imagens adquiridas pelos satélites Landsat 5 e 7, disponíveis em intervalos de 16 dias no catálogo de imagens da Divisão de Geração de Imagens (DGI) do Instituto Nacional de Pesquisas Espaciais (Inpe) (http://www.dgi.Inpe.br/ (DSR/).

Na safra 2002/2003, as imagens do sensor TM não foram gravadas pela estação de recepção do Inpe em Cuiabá, MT, embora o Landsat 5 estivesse operando normalmente nesse período. Os "quick looks" das imagens do sensor ETM+ foram observados somente nas safras de 2000/2001 e 2002/2003, pois, a partir de maio de 2003, o sensor ETM+ começou a apresentar problemas em seu corretor de linha de varredura (Markham et al., 2004) e a estação de recepção do Inpe deixou de gravar esses dados.

O intervalo de avaliação dos "quick looks" em cada ano-safra foi da segunda quinzena de dezembro até a segunda quinzena de março, dividido em sete períodos quinzenais: período 1 , de $16 / 12$ a $31 / 12$; período 2 , de 1\%/1 a 15/1; período 3 , de 16/1 a 31/1; período 4 , de 1/2 a

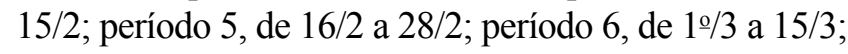
e período 7 , de $16 / 3$ a $31 / 3$. Esta escolha foi feita com base na época de semeadura, que vai de 15 de outubro a 15 de dezembro (Tecnologias..., 2006) e no trabalho de Berka $\&$ Rudorff (2003), que consideram as imagens dos meses de janeiro e fevereiro como sendo as mais indicadas para identificação da soja nas imagens de satélite no Estado do Paraná. Por exemplo, as lavouras de soja semeadas em outubro e início de novembro já cobrem o solo em janeiro e aparecem bem destacadas nas imagens de janeiro; enquanto as lavouras semeadas em fim de novembro e início de dezembro estão bem destacadas nas imagens de fevereiro. Ao passo que as lavouras semeadas no início de outubro já estão senescentes ou até mesmo colhidas em fevereiro do ano seguinte. Desta forma, as áreas de soja de semeadura precoce, média e tardia poderiam ser claramente identificadas nas imagens.

A avaliação dos "quick looks" foi realizada de forma qualitativa levando-se em conta os aspectos: utilidade da imagem para identificação da soja; cobertura de nuvens da imagem; e qualidade da gravação da imagem. Os "quick looks" foram agrupados em sete classes, que estão descritas e exemplificadas na Figura 2.

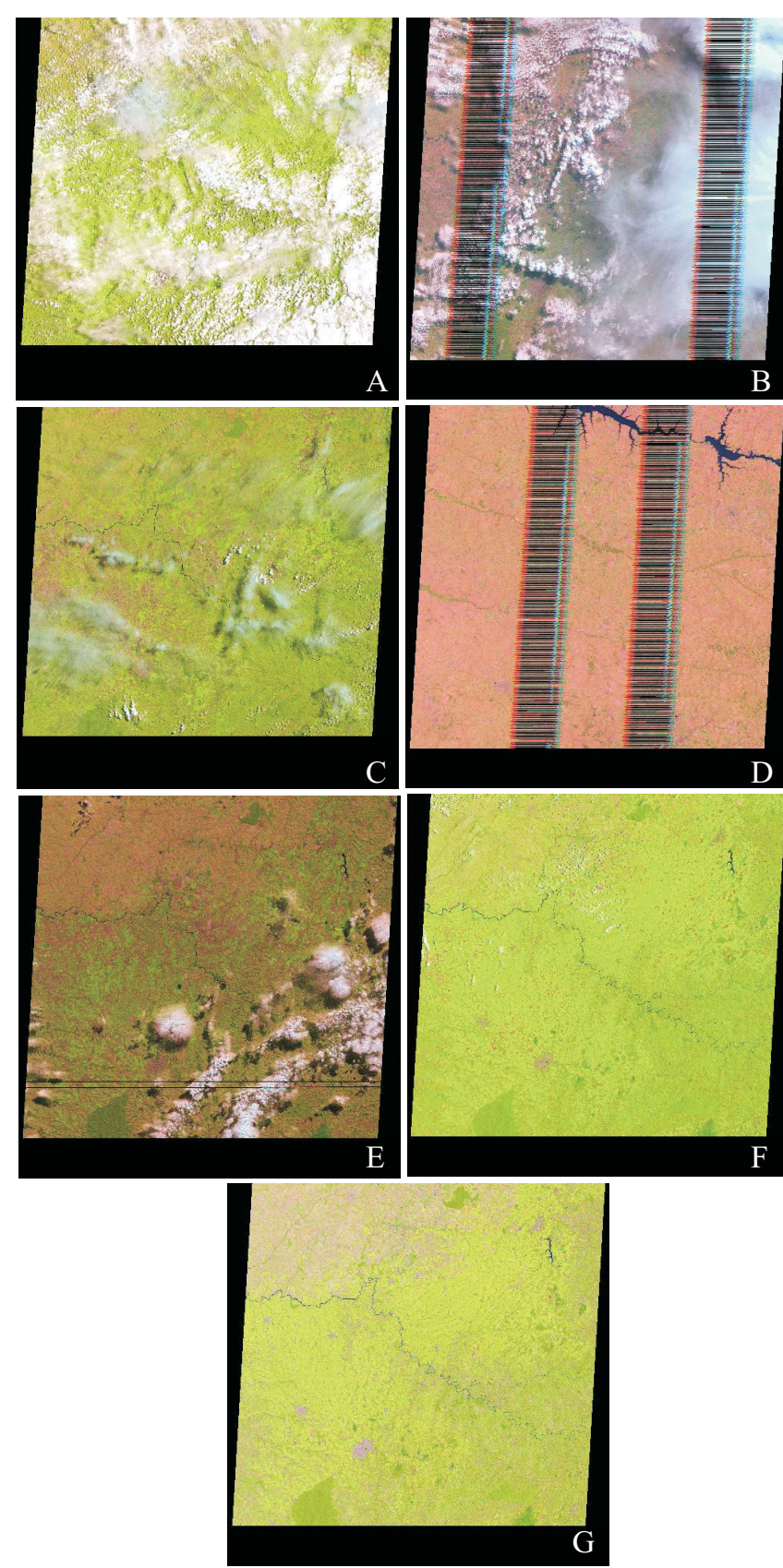

Figura 2. Ilustração dos "quick looks", obtidos das imagens dos satélites Landsat 5 e 7: A, sem utilidade por cobertura de nuvens; $\mathrm{B}$, sem utilidade por cobertura de nuvens e por problemas técnicos; $\mathrm{C}$, útil com presença de nuvens e névoa; $\mathrm{D}$, útil com problemas técnicos; $\mathrm{E}$, útil com nuvens, névoa e problema técnico; F, útil com pouca presença de nuvens e névoa; $\mathrm{G}$, totalmente utilizável. 
A contabilização do número de imagens para cada cena, em cada ano-safra, foi realizada de acordo com a quantidade de "quick looks" que deveria constar no catálogo; a quantidade de "quick looks" que consta no catálogo; e a classificação qualitativa realizada de acordo com a descrição apresentada na Figura 2. Em seguida, foi obtido o somatório do resultado de todas as cenas, por safra, e separados por sensor. Também foi calculado o total por ano-safra e o total geral do período avaliado.

A possibilidade de mapear a área total em cada cena, para cada um dos anos safras, foi verificada por meio da seguinte classificação: $U$, cena totalmente possível de ser mapeada - se duas imagens estiverem totalmente livres de nuvens (Figura $2 \mathrm{G}$ ), nos períodos 2 a 5 (Berka \& Rudorff, 2003; Rizzi \& Rudorff, 2005), ou se pelo menos quatro imagens estiverem parcialmente cobertas por nuvens e/ou com problema técnico (Figura $2 \mathrm{C}, \mathrm{D}, \mathrm{E}$ e F) e se complementarem nos períodos 2 a 5 ; PU, cena parcialmente possível de ser mapeada - se apenas as imagens dos períodos 6 e 7 estiverem totalmente livres de nuvens (Figura $2 \mathrm{G}$ ), ou se as imagens dos períodos 1 a 5 estiverem parcialmente cobertas por nuvens e/ou com problema técnico (Figura $2 \mathrm{C}, \mathrm{D}$, E e F) e não se complementarem; I, cena impossível de ser mapeada - se todas as imagens dos períodos avaliados apresentarem cobertura total de nuvens e/ou com problemas técnicos (Figuras $2 \mathrm{~A}$ e B).

A partir dessa classificação, foi calculada a percentagem de cenas possíveis de serem mapeadas completamente em cada ano-safra, em todo o Estado do Paraná.

\section{Resultados e Discussão}

Do total de "quick looks", que deveriam estar contidos no catálogo da DGI para o período analisado, apenas $43,4 \%$ foram aproveitadas para a identificação e mapeamento da área cultivada de soja (Tabela 1). Dos "quick looks" não aproveitados, foi observado que $41,3 \%$ não estavam disponíveis por problemas técnicos; $57,2 \%$ não foram aproveitados por presença de nuvens (Figura 2 A); e 1,5\% foi inaproveitável por presença de nuvens e problemas técnicos (Figura $2 \mathrm{~B}$ ). Ressalta-se ainda que, dos "quick looks" aproveitáveis, apenas $6,3 \%$ apresentaram $0 \%$ de cobertura de nuvens (Figura $2 \mathrm{G}$ ); 22,8\% apresentaram pouca cobertura de nuvens (Figura 2 F) e 70,9\% são parcialmente utilizáveis, pois apresentaram nuvens e névoa ou problema técnico (Figura $2 \mathrm{C}, \mathrm{D}$ e E).

A quantidade de imagens utilizáveis com $0 \%$ de cobertura de nuvens aumentou ligeiramente ao longo dos sete períodos avaliados (Figura 3). A mesma tendência de aumento foi observada nas imagens disponíveis com pouca cobertura de nuvens (Figura 3), quando agrupadas mensalmente. Sano et al. (2007) também observaram essa tendência de aumento, em que a probabilidade de se obter imagens, com menos de $10 \%$ de cobertura de nuvens no Cerrado brasileiro, aumentou gradativamente no período de dezembro a março, embora, com baixa probabilidade de ocorrência.

Porém, a média de imagens disponíveis por ano é bastante baixa em todos os períodos avaliados (Figura 3), o que dificulta o mapeamento das áreas cultivadas com soja. Em nenhum dos anos safra avaliados seria possível mapear a área total cultivada

Tabela 1. Número de "quick looks" das imagens dos sensores TM e ETM+ avaliados de acordo com a classificação da Figura 2, no período de 16 de dezembro a 31 de março, nos anos-safra de 2000/2001 a 2006/2007.

\begin{tabular}{|c|c|c|c|c|c|c|c|c|c|c|c|}
\hline \multirow[t]{3}{*}{ Imagens } & \multicolumn{7}{|c|}{ TM } & \multicolumn{3}{|c|}{ ETM+ } & \multirow[t]{3}{*}{ Total } \\
\hline & $2000 /$ & $2001 /$ & $2002 /$ & $2003 /$ & $2004 /$ & $2005 /$ & $2006 /$ & $2000 /$ & $2001 /$ & $2002 /$ & \\
\hline & 2001 & 2002 & 2003 & 2004 & 2005 & 2006 & 2007 & 2001 & 2002 & 2003 & \\
\hline Não encontrada no catálogo & 6 & 22 & 98 & 7 & 8 & 48 & 2 & 12 & 11 & 8 & 222 \\
\hline \multicolumn{12}{|l|}{ Encontrada no catálogo } \\
\hline Sem utilidade, pela cobertura de nuvens & 41 & 26 & 0 & 35 & 40 & 21 & 47 & 39 & 22 & 36 & 307 \\
\hline Sem utilidade: nuvens e problema técnico & 1 & 3 & 0 & 1 & 0 & 0 & 0 & 2 & 1 & 0 & 8 \\
\hline Útil com presença de nuvens e névoa & 34 & 25 & 0 & 35 & 27 & 20 & 30 & 34 & 40 & 35 & 280 \\
\hline Útil com problema técnico & 0 & 0 & 0 & 0 & 0 & 0 & 0 & 0 & 0 & 0 & 0 \\
\hline Útil com nuvens, névoa e problema técnico & 4 & 0 & 0 & 1 & 4 & 1 & 1 & 1 & 0 & 0 & 12 \\
\hline Útil com pouca presença de nuvens e névoa & 8 & 13 & 0 & 9 & 12 & 2 & 10 & 7 & 18 & 15 & 94 \\
\hline Totalmente utilizável & 1 & 7 & 0 & 7 & 4 & 0 & 3 & 0 & 3 & 1 & 26 \\
\hline Total & 95 & 96 & 98 & 95 & 95 & 92 & 93 & 95 & 95 & 95 & 949 \\
\hline
\end{tabular}


com soja no Estado do Paraná (Tabela 2), já que a condição estabelecida por Berka \& Rudorff (2003) e Rizzi \& Rudorff (2005) não foi alcançada, mesmo com o uso de imagens que se complementassem nos períodos 2 a 5. Este resultado corrobora as observações de Ju \& Roy (2008), que relataram a dificuldade de obtenção de duas imagens livre de nuvens ao longo de uma mesma estação do ano.

O melhor resultado foi alcançado na safra de 2001/2002, quando os satélites Landsat 5 e 7 operaram de forma conjunta (resolução temporal de oito dias), o que permitiu o mapeamento total em 9 das 14 cenas que cobrem o Estado. Há indícios para afirmar que o aumento da resolução temporal de 16 dias para 8 dias aumenta a disponibilidade de imagens livre de nuvens, mas não o

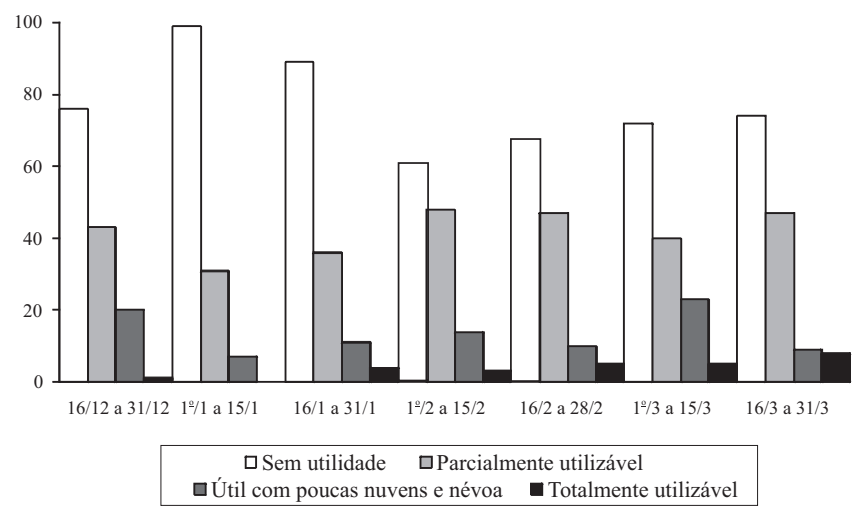

Figura 3. Número de "quick looks" das imagens dos sensores TM e ETM+, agrupados em sete períodos entre 16 de dezembro e 31 de março, nos anos-safra 2000/2001 a 2006/2007. suficiente para mapear a cultura da soja em todo Estado do Paraná.

No Rio Grande do Sul, Rizzi \& Rudorff (2005) mapearam completamente a área de soja do Estado no ano-safra de 2000/2001 por disporem de imagens livres de cobertura de nuvens, em datas favoráveis para a identificação e mapeamento da soja. No entanto, nesse mesmo ano-safra, apenas uma cena seria mapeada completamente no Paraná (Tabela 2). Diante da impossibilidade do mapeamento sistemático da área cultivada com soja no Estado do Paraná por meio de satélites semelhantes ao Landsat, algumas alternativas foram identificadas: a) acréscimo no número de plataformas que operam conjuntamente para aumentar a resolução temporal de imagens de resolução espacial média (Dorigo et al., 2007). Atualmente essa alternativa parece ser uma das mais viáveis para contornar a limitação imposta pela cobertura de nuvens na aquisição de imagens de sensoriamento remoto óptico com qualidade (Wulder et al., 2008b). Um exemplo desse tipo de iniciativa é o "disaster monitoring constellation" (DMC), que é uma constelaçãodecincopequenos satélitescomcargaútilmuito semelhante e que adquirem imagens com características similares às do sensor TM, mas com resolução temporal quase diária (Curiel et al., 2005). A grande limitação do uso das imagens do DMC é o elevado custo envolvido na sua obtenção, o que inviabiliza um projeto operacional para o mapeamento sistemático da cultura da soja. Além disso, a ausência de um sensor que opere na faixa do espectro eletromagnético do infravermelho médio pode prejudicar a identificação da cultura da soja, já que esta

Tabela 2. Qualidade das imagens para identificação e mapeamento da soja nos anos-safra de 2000/2001 a 2006/2007(1).

\begin{tabular}{|c|c|c|c|c|c|c|c|}
\hline \multirow[t]{2}{*}{ Cena } & \multicolumn{7}{|c|}{ Anos-safra } \\
\hline & $2000 / 2001$ & $2001 / 2002$ & $2002 / 2003$ & $2003 / 2004$ & $2004 / 2005$ & $2005 / 2006$ & $2006 / 2007$ \\
\hline $220 / 77$ & $\mathrm{PU}$ & $\mathrm{PU}$ & PU & PU & PU & $\mathrm{I}$ & $\mathrm{PU}$ \\
\hline $220 / 78$ & PU & PU & PU & PU & PU & I & I \\
\hline $221 / 76$ & $\mathrm{U}$ & $\mathrm{U}$ & PU & $\mathrm{U}$ & PU & $\mathrm{I}$ & PU \\
\hline $221 / 77$ & PU & PU & PU & PU & PU & PU & PU \\
\hline $221 / 78$ & PU & PU & PU & PU & PU & $\mathrm{U}$ & PU \\
\hline $222 / 76$ & PU & $\mathrm{U}$ & $\mathrm{PU}$ & $\mathrm{U}$ & PU & PU & PU \\
\hline $222 / 77$ & PU & $\mathrm{U}$ & PU & $\mathrm{U}$ & PU & PU & PU \\
\hline $222 / 78$ & PU & $\mathrm{P}$ & PU & PU & PU & PU & PU \\
\hline $223 / 76$ & PU & $\mathrm{U}$ & PU & PU & PU & PU & $\mathrm{U}$ \\
\hline $223 / 77$ & PU & $\mathrm{U}$ & $\mathrm{U}$ & PU & $\mathrm{PU}$ & $\mathrm{PU}$ & PU \\
\hline $223 / 78$ & PU & $\mathrm{U}$ & $\mathrm{U}$ & PU & $\mathrm{U}$ & $\mathrm{PU}$ & PU \\
\hline $224 / 76$ & PU & $\mathrm{U}$ & PU & $\mathrm{U}$ & PU & PU & PU \\
\hline $224 / 77$ & PU & $\mathrm{U}$ & $\mathrm{PU}$ & $\mathrm{U}$ & $\mathrm{U}$ & PU & $\mathrm{U}$ \\
\hline $224 / 78$ & $\mathrm{PU}$ & $\mathrm{U}$ & PU & $\mathrm{U}$ & $\mathrm{U}$ & $\mathrm{PU}$ & $\mathrm{PU}$ \\
\hline Cenas U $(\%)$ & 7 & 64 & 14 & 43 & 21 & 7 & 14 \\
\hline
\end{tabular}

${ }^{(1)} \mathrm{U}$, totalmente possível de ser mapeada; PU, cena parcialmente possível de ser mapeada; I, cena impossível de ser mapeada. 
faixa é importante para distinguir as respostas espectrais apresentadas por diferentes espécies vegetais (Cox, 1983); b) utilização de sensores que possuam resolução espacial moderada e resolução temporal quase diária, como é o caso do sensor MODIS. Este foi um avanço observado a partir de 2000, quando as imagens de resolução temporal quase diária tiveram a resolução espacial elevada de baixa $(1 \mathrm{~km}$ ou 100 ha; e.g. AVHRR; Vegetation) para moderada ( $250 \mathrm{~m}$ ou 6,25 ha; e.g. MODIS; MERIS). Apesar da adequada resolução temporal no mapeamento da área cultivada de soja (Lamparelli et al., 2008), a resolução espacial moderada das imagens do sensor MODIS dificulta a identificação precisa das lavouras agrícolas, particularmente, em regiões de minifúndios, onde as áreas de soja são muito fragmentadas (Rudorff et al., 2007; Wardlow \& Egbert, 2008); c) utilização de imagens obtidas por diferentes satélites e/ou sensores para aumentar a resolução temporal das imagens. No entanto, Wulder et al. (2008a) comenta que uma das desvantagens do uso de dados oriundos de diferentes satélites e/ou sensores é o aumento do custo com a aquisição de imagens, o que pode inviabilizar um projeto de natureza operacional.

O Brasil pretende se capacitar para receber, a partir de 2009, imagens do satélite Resourcesat (IRS-P6), que tem a bordo um sensor denominado AWiFS com resolução espacial de $56 \mathrm{~m}$ e resolução temporal de 5 dias. As imagens desse sensor irão trazer benefícios a diversas aplicações ligadas ao setor agrícola no Brasil, inclusive a possibilidade de se mapear sistematicamente a cultura da soja, para fins de estimativa de área plantada não só no Estado do Paraná, mas para todo o Brasil.

O futuro do SR óptico, para fins de obtenção de informações agrícolas, depende de alternativas que aumentem significativamente a resolução temporal das imagens de resolução espacial média.

\section{Conclusões}

1. A presença de nuvens nas imagens obtidas por sensores ópticos deve ser levada em conta no mapeamento sistemático das áreas cultivadas com culturas de verão, no Brasil.

2. A resolução temporal de 16 dias, dos satélites Landsat 5 e 7 , ou mesmo de 8 dias, quando esses satélites operaram em conjunto, é insuficiente para realizar o mapeamento sistemático da área cultivada com soja no Estado do Paraná.

\section{Agradecimentos}

Ao Dr. Antônio Roberto Formaggio e ao Dr. Yosio Edemir Shimabukuro, pelas valiosas sugestões.

\section{Referências}

ASNER, G.P. Cloud cover in Landsat observations of the Brazilian Amazon. International Journal of Remote Sensing, v.22, p.3855-3862, 2001.

BERKA, L.M.S.; RUDORFF, B.F.T. Estimativa de área plantada com soja através de imagens Landsat em municípios do norte do Paraná. In: SIMPÓSIO BRASILEIRO DE SENSORIAMENTO REMOTO (SBSR), 11., 2003, Belo Horizonte. Anais... São José dos Campos: Inpe, 2003. p.27-31. 1 CD-ROM. Disponível em: $<$ http://marte.dpi.Inpe.br/col/ltid.Inpe.br/sbsr/2002/11.18.18.36/ doc/01_469.pdf>. Acesso em: 17 jul. 2008.

COX, S.C. (Ed.). The multispectral imaging science working group: final report. Washington: Nasa, 1983. (Nasa Conference Publication, 2260).

CURIEL, A.S.; BOLAND, L.; COOKSLEY, J.; BEKHTI, M.; STEPHENS, P.; SUN, W.; SWEETING, M. First results from the disaster monitoring constellation (DMC). Acta Astronautica, v.56, p.261-271, 2005.

DORAISWAMY, P.C.; HATFIELD, J.L.; JACKSON, T.J.; AKHMEDOV, B.; PRUEGER, J.; STERN, A. Crop condition and yield simulations using Landsat and MODIS. Remote Sensing of Environment, v.92, p.548-559, 2004.

DORIGO, W.A.; ZURITA-MILLA, R.; DE WIT, A.J.W.; BRAZILE, J.; SINGH, R.; SCHAEPMAN, M.E. A review on reflective remote sensing and data assimilation techniques for enhanced agroecosystem modeling. International Journal of Applied Earth Observation and Geoinformation, v.9, p.165-193, 2007.

FONTANA, D.C.; WEBER, E.; DUCATI, J.; FIGUEIREDO, D.C.; BERLATO, M.A.; BERGAMASCHI, H. Monitoramento e previsão de safras no Brasil. In: SIMPOSIO LATINOAMERICANO DE PERCEPCIÓN REMOTA Y SISTEMAS DE INFORMACIÓN ESPACIAL (SELPER), 9., 2000, Puerto Iguazú. Anais... Puerto Iguazú: SELPER, 2000. p.87-96. Disponível em: $<$ http://www. selper.org/trabajos/agr016.pdf>. Acesso em: 25 out. 2008.

IBGE. Malha Municipal Digital 2005. Rio de Janeiro, 2007. Mapas. Escala 1:500.000. Disponível em: <ftp://geoftp.ibge.gov. br/mapas/malhas_digitais/municipio_2005/E500/Proj_Policonica/ ArcView_shp/Uf/>. Acesso em: 12 ago. 2007a.

IBGE. Produção Agrícola Municipal. Disponível em: $<\mathrm{http}: / /$ www.sidra.ibge.gov.br/bda/pesquisas/pam/default.asp $>$. Acesso em: 27 ago. $2007 \mathrm{~b}$.

IPPOLITI-RAMILO, G.A.; EPIPHANIO, J.C.N.; SHIMABUKURO, Y.E. Landsat-5 Thematic Mapper data for pre-planting crop area evaluation in tropical countries. International Journal of Remote Sensing, v.24, p.1521-1534, 2003.

JU, J.; ROY, D.P. The availability of cloud-free Landsat ETM+ data over the conterminous United States and globally. Remote Sensing of Environment, v.112, p.1196-1211, 2008.

JUSTICE, C.O.; TOWNSHEND, J.R.G. Special issue on the Moderate Resolution Imaging Spectroradiometer (MODIS): a new generation of land surface monitoring. Remote Sensing of Environment, v.83, p.1-2, 2002. 
LAMPARELLI, R.A.C.; CARVALHO, W.M.O.; MERCANTE, E. Mapeamento de semeaduras de soja (Glycine max (L.) Merr.) mediante dados MODIS/Terra e TM/Landsat 5: um comparativo. Engenharia Agrícola, v.28, p.334-344, 2008.

MARKHAM, B.L.; STOREY, J.C.; WILLIAMS, D.L.; IRONS, J.R. Landsat sensor performance: history and current status. IEEE Transactions on Geoscience and Remote Sensing, v.42, p.2691-2694, 2004.

MAXWELL, S.K.; NUCKOLS, J.R.; WARD, M.H.; HOFFER, R.M. An automated approach to mapping corn from Landsat imagery. Computers and Electronics in Agriculture, v.43, p.43-54, 2004.

MENDONÇA, F.J.; LEE, D.C.L.; TARDIN, A.T.; CHEN, S.C.; NOVAES, R.A.; SHIMABUKURO, Y.E. Resultados significantes do projeto estatísticas agrícolas: 1975-1978. São José dos Campos: Inpe, 1979. 29p. (Inpe-1609-NTE/155).

OETTER, D.R.; COHEN, W.B.; BERTERRETCHE, M.; MAIERSPERGER, T.K.; KENNEDY, R.E. Land cover mapping in an agricultural setting using multiseasonal Thematic Mapper data. Remote Sensing of Environment, v.76, p.139-155, 2000.

PARANÁ. Secretaria de Estado da Agricultura e do Abastecimento (SEAB). Instituto de Terras, Cartografia e Florestas (ITCF). Atlas do Estado do Paraná. Curitiba, 1987.

RIZZI, R.; RUDORFF, B.F.T. Estimativa da área de soja no Rio Grande do Sul por meio de imagens Landsat. Revista Brasileira de Cartografia, v.57, p.226-234, 2005.

RUDORFF, C.M.; RIZZI, R.; RUDORFF, B.F.T.; SUGAWARA, L.M.; VIEIRA, C.A.O. Superficies de resposta espectro-temporal de imagens do sensor MODIS para classificação de área de soja no Estado do Rio Grande do Sul. Ciência Rural, v.37, p.118-125, 2007.
RUDORFF, B.F.T.; SUGAWARA, L.M. Mapeamento da cana-de-açúcar na região Centro-Sul via imagens de satélites. Informe Agropecuário, v.28, p.79-86, 2007.

SANO, E.E.; FERREIRA, L.G.; ASNER, G.P.; STEINKE, E.T. Spatial and temporal probabilities of obtaining cloud-free Landsat images over the Brazilian tropical savanna. International Journal of Remote Sensing, v.28, p.2739-2752, 2007.

TECNOLOGIAS de produção de soja: Paraná 2007. Londrina: Embrapa Soja, 2006. 217p.

UNITED STATES GEOLOGICAL SURVEY (USGS). WRS Scene Center and Corner Points. Disponível em: $<$ http://landsat. usgs.gov/tools_WRScornerPoints.xls>. Acesso em: 17 jul. 2008.

VAN NIEL, T.G.; MCVICAR, T.R. Determining temporal windows for crop discrimination with remote sensing: a case study in south-eastern Australia. Computers and Electronics in Agriculture, v.45, p.91-108, 2004.

WARDLOW, B.D.; EGBERT, S.L. Large-area crop mapping using time-series MODIS $250 \mathrm{~m}$ NDVI data: an assessment for the U.S. Central Great Plains. Remote Sensing of Environment, v.112, p.1096-1116, 2008.

WULDER, M.A.; BUTSON, C.R.; WHITE, J.C. Cross-sensor change detection over a forested landscape: options to enable continuity of medium spatial resolution measures. Remote Sensing of Environment, v.112, p.796-809, 2008a.

WULDER, M.A.; WHITE, J.C.; GOWARD, S.N.; MASEK, J.G.; IRONS, J.R.; HEROLD, M.; COHEN, W.B.; LOVELAND, T.R.; WOODCOCK, C.E. Landsat continuity: issues and opportunities for land cover monitoring. Remote Sensing of Environment, v.112, p.955-969, 2008b.

Recebido em 17 de julho de 2008 e aprovado em 28 de novembro de 2008 